\title{
Articulo Original / Original Article \\ New sources of botanical acaricides from species of Croton with potential use in the integrated management of Tetranychus urticae
}

\author{
[Nuevas fuentes de acaricidas botánicos de especies de Croton con uso potencial \\ en el manejo integrado de Tetranychus urticae]
}

\author{
Claudio AG da Camara ${ }^{1}$, Carolina A de Araujo ${ }^{1}$, Marcilio M de Moraes ${ }^{1}$, \\ João PR de Melo $^{2}$ \& Maria FA Lucena ${ }^{3}$ \\ ${ }^{1}$ Department of Chemistry, Federal Rural University of Pernambuco, Recife, PE, Brazil \\ ${ }^{2}$ Department of Agronomy, Federal Rural University of Pernambuco, Recife, PE, Brazil \\ ${ }^{3}$ Rural Health and Technology Center, Federal University of Campina Grande, Patos, PB, Brazil
}

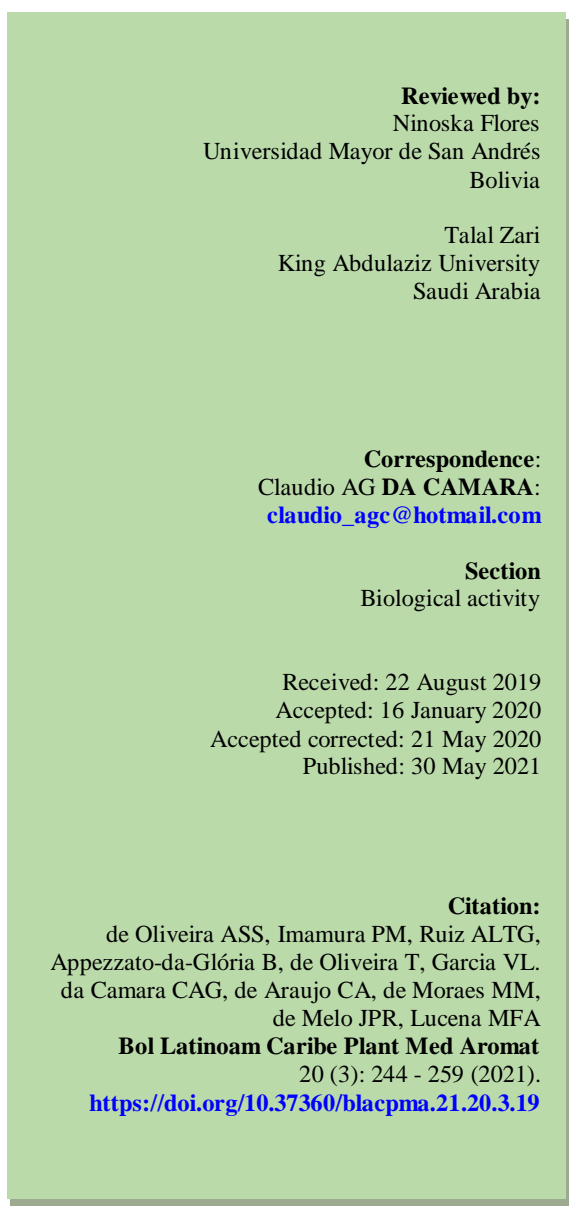

Abstract: Essential oils from the leaves and stems of Croton adenocalyx, C. grewioides, C. heliotropiifolius and C. blanchetianus obtained through hydrodistillation were analyzed by GC-MS. We then investigated the lethal and sublethal effects of the Croton oils and 15 major constituents against Tetranychus urticae. $\beta$-Caryophyllene was the major component in the leaf and stem oils from $C$. heliotropiifolius and C. adenocalyx. Spathulenol and (E)-anethole were the major constituents identified in the leaf and stem oils of $C$. blanchetianus and $C$. grewioides, respectively. The oil with the greatest lethal and sublethal effects was those from $C$. adenocalyx. Among the constituents $\beta$-caryophyllene and spatulenol were the most toxic to the mite, whereas eugenol and methyl eugenol were the most repellent. The toxicity and repellency of the Croton oils, particularly the oils from C. adenocalyx, demonstrate that these oils constitute a promising alternative to synthetic acaricides for use in the control of T. urticae.

Keywords: Croton ssp.; Essential oil; Botanical acaricide; Two-spotted spider mite.

Resumen: Los aceites esenciales de las hojas y tallos de Croton adenocalyx, C. growioides, $C$. heliotropiifolius y $C$. blanchetianus obtenidos mediante hidrodestilación fueron analizados a través de GC-MS. Se investigaron los efectos letales y subletales de los aceites de Croton y 15 componentes principales contra Tetranychus urticae. El $\beta$-cariofileno fue el componente principal en los aceites de hojas y tallos de C. heliotropiifolius y $C$. adenocalyx. El espatulenol y el (E)-anetol fueron los principales componentes identificados en los aceites de hojas y tallos de C. blanchetianus y $C$. growioides, respectivamente. El aceite con los mayores efectos letales y subletales fue el de C. adenocalyx. Entre los componentes, el $\beta$-cariofileno y el espatulenol fueron los más tóxicos para el ácaro, mientras que el eugenol y el metil eugenol fueron los más repelentes. La toxicidad y la repelencia de los aceites de Croton, particularmente los aceites de $C$. adenocalyx, demuestran que estos aceites constituyen una alternativa prometedora respecto a los acaricidas sintéticos para uso en el control de T. urticae.

Palabras clave: Croton ssp.; Aceite esencial; Acaricida botánico; Ácaro araña con dos manchas. 


\section{INTRODUCTION}

According to the reports of farmers in the main family farming niches in the semiarid region of the state of Pernambuco, Brazil, significant losses of tomato and cucumber crops in protected environments are common due to the attack of several types of pests, particularly the two-spotted spider mite (Tetranychus urticae Kock). The main form of controlling this pest is the use of commercial acaricides, such as Ortus $^{\circledR}$. However, the indiscriminate use of these products has led to populations of $T$. urticae resistant to the active ingredients (Van Leeuwen et al., 2010). A low-cost alternative that causes less harm to the ecosystem than these synthetic acaricides is the use of formulations containing natural products derived from plants, such as essential oils.

Among the plants with broad occurrence in the Caatinga biome of northeast Brazil, specie of the genus Croton L stand out due to their previously investigated acaricidal potential (Neves \& da Camara, 2011; da Camara et al., 2017). This genus belongs to the subfamily Crotonoideae and comprises around 1300 species of herbs, shrubs and trees distributed in tropical and subtropical regions throughout the world (Berry et al., 2005). Among the 350 species of Croton recorded for Brazil (Van Ee et al., 2011), 35 are found in Pernambuco, where some are considered endemic (Silva et al., 2010). Plants of this genus are sources of bioactive compounds with a variety of biological properties, including insecticidal (Cruz et al., 2017; Silva et al., 2018) and acaricidal (da Camara et al., 2017; Castro et al., 2019) activity, which are attributed to secondary metabolites pertaining to the diterpene, alkaloid and terpene classes, the latter of which are found in essential oils.

The species $C$. adenocalyx, $C$. grewioides, $C$. heliotropiifolius and $C$. blanchetianus are native to the semiarid region of the state of Pernambuco and are broadly distributed around agricultural niches in the region, used by local communities in the form of tea for the treatment of gastrointestinal diseases and to relieve headache or fever (Hiruma-Lima et al., 2000; Macêdo et al., 2015). There are no previous reports in the literature on the biological activity of the essential oil from Croton adenocalyx. Although a previous investigation evaluated the antimicrobial, antinociceptive and insecticidal action of $C$. grewioides, C. heliotropiifolius and C. blanchetianus, no studies have addressed the acaricidal effect of the essential oils from these species on T. urticae.

Employing the acaricidal properties of aromatic flora for the preparation of more environmentally benign and less harmful formulations could be a viable approach for use in the control of T. urticae within the concept of integrated pest management. Thus, the aim of the present study was to investigate whether the essential oils from the leaves and stems of $C$. adenocalyx, $C$. grewioides, $C$. heliotropiifolius and $C$. blanchetianus have effects on $T$. urticae through acaricidal and repellant mechanisms. We also investigated the acaricidal properties of 15 chemical compounds identified as the major constituents of the essential oils. The results were compared to those achieved with the commercial acaricides Ortus ${ }^{\circledR}$ and Azamax ${ }^{\circledR}$ used as positive controls.

\section{MATERIAL AND METHODS Collection of plant material}

The fresh leaves and stems of Croton adenocalyx Baill, C. grewioides Baill, C. heliotropiifolius Kunth and $C$. blanchetianus Baill were collected in the Vale do Catimbau, a Brazilian national park in the state of Pernambuco-Brazil. The plants were identified by Botanist Dra. Maria F.A. Lucena. Voucher of both samples were mounted and deposited in the herbarium of the Federal University of Pernambuco, under number: (30491) C. heliotropiifolius, (49390) C. adenocalyx, and herbarium of the Federal Rural University of Pernambuco, under number: (48219) $C$. blanchetianus and (42193) C. grewioides.

\section{Isolation of Essential Oils}

Essential oils from the leaves and stems $(100 \mathrm{~g})$ of $C$. adenocalyx, C. blanchetianus, $C$. grewioides and $C$. heliotropiifolius were obtained by hydrodistillation using a modified Clevenger apparatus for $4 \mathrm{~h}$. The oil layers were separated and dried over anhydrous sodium sulfate, stored in hermetically sealed glass containers and kept at a low temperature $\left(-5^{\circ} \mathrm{C}\right)$ until the bioassays and analyses. Total oil yields were expressed as percentages $(\mathrm{g} / 100 \mathrm{~g}$ of fresh plant material). All experiments were carried out in triplicate.

\section{Chemicals}

The constituents used as standards in the identification of volatile compounds in the oils were purchased from Sigma-Aldrich (Brazil). Monoterpenes ( $\alpha$-pinene, $\beta$-pinene, $p$-cymene, 1,8 cineole, camphor, $\beta$-phellandrene, $\alpha$-phellandrene), sesquiterpenes ( $\beta$-caryophyllene, bicyclogermacrene, spathulenol and caryophyllene oxide) and phenylpropanoid (methyl eugenol, eugenol, (E)anethole, eugenyl acetate) were selected for the 
bioassays due to the fact that these compounds were identified in the Croton oils as a major constituents and are available commercially. Fenpyroximate (Ortus ${ }^{\circledR} 50$ g i.a./L SC Arysta Lifescience) and azadirachtin (Azamax ${ }^{\circledR} 12$ g i.a./L EC E.I.D. Parry) were acquired from the local market and used as positive controls.

\section{Gas chromatography FID analysis}

Gas Chromatography (GC) identification was performed using a Hewlett-Packard 5890 Series II GC apparatus equipped with a flame ionization detector (FID) and a non-polar DB-5 fused silica capillary column $(30 \mathrm{~m} \times 0.25 \mathrm{~mm} \times 0.25 \mu \mathrm{m})(\mathrm{J}$ and W Scientific). The oven temperature was programmed from 60 to $240^{\circ} \mathrm{C}$ at a rate $3^{\circ} \mathrm{C} / \mathrm{min}$. Injector and detector temperatures were $260^{\circ} \mathrm{C}$. Hydrogen was used as the carrier gas at a flow rate of $1 \mathrm{~mL} / \mathrm{min}$ in split mode (1:30). The injection volume was $0.5 \mu \mathrm{L}$ of diluted solution $(1 / 100)$ of oil in $n$ hexane. The percentage of each compound was obtained from GC-FID peak areas in the order of the DB-5 column elution and expressed as the relative percentage of the area of the chromatograms. The analysis was conducted in triplicate.

\section{Gas chromatography-mass spectrometry analysis}

The GC-MS analysis of the essential oils was carried out using a Varian 220-MS IT GC system with a mass selective detector, mass spectrometer in EI 70 $\mathrm{eV}$ with a scan interval of $0.5 \mathrm{~s}$ and fragments from 40 to $550 \mathrm{Da}$. fitted with the same column and temperature program as that for the GC-FID experiments, with the following parameters: carrier gas $=$ helium; flow rate $=1 \mathrm{~mL} / \mathrm{min}$; split mode (1:30); injected volume $=1 \mu \mathrm{L}$ of diluted solution $(1 / 100)$ of oil in n-hexane.

\section{Identification of components}

Identification of the components was based on GCMS retention indices with reference to a homologous series of $\mathrm{C}_{8}-\mathrm{C}_{40} \mathrm{n}$-alkanes calculated using the Van Den Dool and Kratz equation (Van Den Dool \& Kratz, 1963) and by computer matching against the mass spectral library of the GC-MS data system (NIST version 14) and co-injection with authentic standards as well as other published mass spectra (Adams, 2017). Area percentages were obtained from the GC-FID response without the use of an internal standard or correction factors.

\section{Acquisition and rearing Tetranychus urticae.}

Specimens of Tetranychus urticae (Acari:
Tetranychidae) was originally collected from grapevine (Vitis vinifera L.) in the municipality of Petrolina-PE $\left(09^{\circ} 12^{\prime} 43^{\prime \prime} \mathrm{S}\right.$; 40 $\left.29^{\prime} 12^{\prime \prime} \mathrm{W}\right)$, and since then maintained in laboratory at the Agronomy Department of the Federal Rural University of Pernambuco, Brazil. T. urticae were rearing at a temperature of $25 \pm 1^{\circ} \mathrm{C}$, relative humidity of $65 \pm$ $5 \%$ and a $12 \mathrm{~h}$ photoperiod and without any exposure to acaricides. The breeding method was adapted from Ribeiro et al. (2016).

\section{Contact assay}

Leaf disc painting method described by Moraes et al. (2012) was used to test the action of Croton oils and positive control by contact toxicity. Experiments were performed with open Petri dishes $(10 \mathrm{~cm}$ diameter). Leaf discs $(2.5 \mathrm{~cm}$ diameter) were cut from leaves of greenhouse-grown jack bean. The concentrations of Croton ranged from 0.10 to 2.50 $\mu \mathrm{L} / \mathrm{mL}$ (leaves) and 0.50 to $4.50 \mu \mathrm{L} / \mathrm{ml}$ (stems) for $C$. adenocalyx, 10.0 to $100 \mu \mathrm{L} / \mathrm{mL}$ (leaves and stems) for $C$. heliotropiifolius; 1.00 to $8.00 \mu \mathrm{L} / \mathrm{mL}$ (leaves) and 5.0 to $40.0 \mu \mathrm{L} / \mathrm{mL}$ (stems) for $C$. blanchetianus; 5.00 to $40.0 \mu \mathrm{L} / \mathrm{mL}$ (leaves) and 10.0 to $80 \mu \mathrm{L} / \mathrm{mL}$ (stems) for $C$. grewioides. The concentration of botanical and conventional insecticides used as positive control vary from 0.05 to $0.80 \mu \mathrm{L} / \mathrm{mL}$ for Azamax ${ }^{\circledR}$ and from 0.01 to $0.40 \mu \mathrm{L} / \mathrm{mL}$ for Ortus ${ }^{\circledR} 50$ SC. A $20 \mu \mathrm{L}$ aliquot of each concentration was painted on the underside of the disc with a micropipette. After drying at room temperature for 2 min, each disc was individually placed in the bottom of a Petri dish atop, a $10 \mathrm{~cm}$ diameter disc of filter paper wetted with distilled water. Five adult female mites were introduced into each Petri dish. Mortality was determined under a dissecting microscope $24 \mathrm{~h}$ after the treatment. All mites were considered dead if appendages did not move when prodded with a fine paintbrush. Control mites were held on leaf discs painted with the carrier solvent alone. All treatments were replicated five times. Mortality was recorded and submitted to Probit analysis. To determine $\mathrm{LC}_{50}$ determination with 95 percent confidence level.

\section{Repellency assay}

The repellent effect of the Croton oils were evaluated using area preference method described by Nascimento et al. (2012). Test areas consisted of disks of pig bean leaves ( $C$. ensiformis) cut in half $\left(9.81 \mathrm{~cm}^{2}\right)$. Test solutions were prepared by diluting $10 \mathrm{mg}$ of Croton oils or Ortus ${ }^{\circledR} 50 \mathrm{SC}$ or Azamax $^{\circledR}$ as a positive control in $2 \mathrm{~mL}$ water plus $0.1 \%$ of spreader sticker adjuvante (Tween-80). The 
concentrations of Croton ranged from 0.02 to 0.50 $\mu \mathrm{L} / \mathrm{mL}$ (leaves and stems) for $C$. adenocalyx, 1.0 to $25.0 \mu \mathrm{L} / \mathrm{mL}$ (leaves) and 1.0 to $50.0 \mu \mathrm{L} / \mathrm{mL}$ (stems) for $C$. heliotropiifolius; 0.05 to $0.50 \mu \mathrm{L} / \mathrm{mL}$ (leaves) and 0.50 to $4.0 \mu \mathrm{L} / \mathrm{mL}$ (stems) for C. blanchetianus; 1.00 to $13.0 \mu \mathrm{L} / \mathrm{mL}$ (leaves and stems) for $C$. grewioides. While the concentration Azamax ${ }^{\circledR}$, insecticides used as positive control were ranged from 0.01 to $0.30 \mu \mathrm{L} / \mathrm{mL}$. Each concentration was uniformly applied to a half-leaf disk using a micropipette. The other half leaf was treated with ethanol alone and used as control. Treated and control disks were air-dried for $10 \mathrm{~min}$ to evaporate the solvent completely. Each disk was placed into a $10 \mathrm{~cm}$ Petri dish. Ten adult mites were released at the center of the disk and the Petri dishes were covered. The treatments were replicated 15 times. The number of mites on the control and treated areas of the disks was recorded after $2 \mathrm{~h}$. To estimate the concentration that repelled $50 \%$ and $90 \%$ of the mites $\left(\mathrm{RC}_{50}\right)$ exposed to each oil, the number of mites on the control and treated areas was recorded and submitted to probit analysis.

\section{Statistical analysis}

The mortality and repellence data were subjected to Probit analysis at $p>0.05$ using PROC PROBIT (Finney, 1971). The concentrations used were calculated based on the logarithmic series proposed by Robertson et al. (2017).

\section{RESULTS}

Hydrodistillation of the different parts of $C$. heliotropiifolius, C. adenocalyx, C. blanchetianus and $C$. grewioides produced yellowish oils with a pleasant aroma. The greatest yields were achieved with the oils from the leaves and stems of $C$. grewioides (leaf oil $=2.40 \pm 0.02 \%$; stem oil $=0.10 \pm$ $0.01 \%$ ), followed by $C$. blanchetianus (leaf oil $=0.50$ $\pm 0.03 \%$; stem oil $=0.03 \pm 0.00 \%), C$. adenocalyx (leaf oil $=0.36 \pm 0.01 \%$; stem oil $=0.01 \pm 0.00 \%$ ) and $C$. heliotropiifolius (leaf oil $=0.21 \pm 0.02 \%$; stem oil $=0.01 \pm 0.00 \%$ ).

GC-MS analysis of the oils enabled the identification of 94 compounds (Table 1). The composition of the $C$. heliotropiifolius (leaf oil = $91.79 \pm 0.44 \%$; stem oil $=87.76 \pm 0.87 \%), C$. adenocalyx (leaf oil $=90.22 \pm 0.73 \%$; stem oil $=$ $91.64 \pm 0.76 \%$ ) and $C$. blanchetianus (leaf oil = $90.56 \pm 0.93 \%$; stem oil $=97.93 \pm 0.78 \%$ ) oils predominantly consisted of sesquiterpenes. In contrast, the leaf and stem oils from $C$. grewioides had higher percentages of phenylpropanoids $(75.7 \pm$ $0.48 \%$ and $75.8 \pm 0.41 \%$, respectively).

Table No. 1A

Percentage compositions of the essential oils from Croton species

\begin{tabular}{|c|c|c|c|c|c|}
\hline \multirow{3}{*}{ Compound } & \multirow{2}{*}{$\mathbf{R I}^{\mathrm{a}}$} & \multicolumn{2}{|c|}{$\begin{array}{c}\text { C. heliotropiifolius } \\
\left(\% \pm S E^{b}\right)\end{array}$} & \multicolumn{2}{|c|}{$\begin{array}{c}\text { C. adenocalyx } \\
\left(\% \pm \mathrm{SE}^{\mathrm{b}}\right)\end{array}$} \\
\hline & & Leaves & Stems & Leaves & Stems \\
\hline & Yield \% & $0.21 \pm 0.02$ & $0.01 \pm 0.00$ & $0.36 \pm 0.01$ & $0.01 \pm 0.00$ \\
\hline$\alpha$-Pinene ${ }^{c}$ & 929 & - & - & $4.65 \pm 0.09$ & $0.25 \pm 0.00$ \\
\hline b-Pinene ${ }^{c}$ & 973 & - & - & $3.12 \pm 0.10$ & $1.38 \pm 0.11$ \\
\hline$\delta$-3-Carene ${ }^{d}$ & 1005 & - & - & - & - \\
\hline$o$-Cymene ${ }^{\mathrm{d}}$ & 1022 & $1.23 \pm 0.01$ & $2.10 \pm 0.07$ & & \\
\hline Limonene $^{c}$ & 1021 & - & - & - & $0.24 \pm 0.00$ \\
\hline 1,8 -Cineole ${ }^{c}$ & 1031 & - & - & 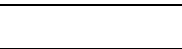 & \\
\hline Linalool $^{\mathrm{c}}$ & 1092 & - & - & - & $0.53 \pm 0.00$ \\
\hline trans-Sabinene hydrate $^{d}$ & 1098 & - & - & - & $0.81 \pm 0.00$ \\
\hline Myrcenol $^{\mathrm{d}}$ & 1113 & - & - & - & $2.64 \pm 0.03$ \\
\hline Camphor $^{\mathrm{C}}$ & 1144 & - & - & - & - \\
\hline Isoborneol $^{d}$ & 1156 & - & $3.34 \pm 0.11$ & - & - \\
\hline$\alpha$-Terpineol ${ }^{d}$ & 1192 & - & - & - & - \\
\hline Methyl chavicol $^{d}$ & 1196 & - & - & - & - \\
\hline$p$-Anisaldehyde ${ }^{d}$ & 1250 & - & - & - & - \\
\hline (Z)-Anethole ${ }^{d}$ & 1251 & - & - & - & - \\
\hline$(E)$-Anethole ${ }^{d}$ & 1280 & - & - & - & - \\
\hline Bornyl acetate ${ }^{d}$ & 1285 & - & $0.91 \pm 0.01$ & - & - \\
\hline$\delta$-Elemene ${ }^{\mathrm{d}}$ & 1330 & - & - & - & - \\
\hline
\end{tabular}




\begin{tabular}{|c|c|c|c|c|c|}
\hline$\alpha$-Cubebene $^{d}$ & 1341 & - & - & - & - \\
\hline$\alpha$-Copaene $^{\mathrm{d}}$ & 1369 & - & - & - & - \\
\hline 3,4-Dihydro-coumarin ${ }^{d}$ & 1376 & - & $3.51 \pm 0.15$ & - & - \\
\hline (Z)-6-Damascenone ${ }^{d}$ & 1381 & - & - & - & - \\
\hline B-Cubebene ${ }^{d}$ & 1383 & - & - & $1.66 \pm 0.10$ & $1.13 \pm 0.09$ \\
\hline B-Bourbonene ${ }^{d}$ & 1384 & - & - & $1.72 \pm 0.17$ & $0.43 \pm 0.00$ \\
\hline iso-Longifolene $\mathrm{d}^{\mathrm{d}}$ & 1385 & - & - & - & $3.23 \pm 0.11$ \\
\hline 6-Elemene ${ }^{\mathrm{d}}$ & 1389 & $6.81 \pm 0.15$ & $17.28 \pm 079$ & $9.34 \pm 0.34$ & $3.46 \pm 0.13$ \\
\hline Cyperene $^{d}$ & 1398 & - & $1.36 \pm 0.10$ & - & - \\
\hline Methyl eugenol $^{\mathrm{C}}$ & 1401 & - & - & - & - \\
\hline B-Caryophyllene ${ }^{c}$ & 1414 & $20.82 \pm 0.38$ & $8.05 \pm 0.22$ & $15.64 \pm 0.62$ & $12.21 \pm 0.72$ \\
\hline b-Cedrene ${ }^{\mathrm{d}}$ & 1416 & - & - & $0.57 \pm 0.01$ & $0.55 \pm 0.01$ \\
\hline b-Ylangene ${ }^{d}$ & 1417 & - & - & $0.52 \pm 0.00$ & $0.53 \pm 0.00$ \\
\hline B-Duprezianene ${ }^{d}$ & 1418 & - & - & $0.58 \pm 0.00$ & $0.48 \pm 0.00$ \\
\hline Lavandulyl isobutanoate $^{d}$ & 1419 & - & - & - & $1.05 \pm 0.01$ \\
\hline Linalol butanoate $^{\mathrm{d}}$ & 1421 & - & - & $5.00 \pm 0.20$ & $5.13 \pm 0.14$ \\
\hline Dictamnol $^{d}$ & 1428 & - & - & $1.48 \pm 0.05$ & $0.77 \pm 0.02$ \\
\hline cis-Thujupsene $^{\mathrm{d}}$ & 1429 & - & - & $0.31 \pm 0.02$ & - \\
\hline b-Copaene ${ }^{d}$ & 1429 & - & - & $11.45 \pm 0.32$ & $8.45 \pm 0.28$ \\
\hline B-Gurjunene ${ }^{d}$ & 1430 & - & - & $1.40 \pm 0.03$ & $6.27 \pm 0.20$ \\
\hline trans- $\alpha$-Bergamotene ${ }^{d}$ & 1430 & - & - & - & - \\
\hline$\gamma$-Elemene ${ }^{\mathrm{d}}$ & 1432 & $0.93 \pm 0.00$ & - & $14.80 \pm 0.84$ & $6.85 \pm 0.12$ \\
\hline B-Dihydro-ionone ${ }^{d}$ & 1434 & - & - & $3.44 \pm 0.07$ & $0.87 \pm 0.02$ \\
\hline$\alpha$-Guaiene ${ }^{d}$ & 1436 & - & - & $0.71 \pm 0.01$ & $0.57 \pm 0.05$ \\
\hline Aromadendrene $^{c}$ & 1436 & - & - & $1.90 \pm 0.14$ & $2.91 \pm 0.12$ \\
\hline$\alpha$-Himachalene ${ }^{d}$ & 1446 & - & - & - & - \\
\hline neo- $\alpha$-Clovene ${ }^{d}$ & 1448 & - & - & - & - \\
\hline$\alpha$-Humulene ${ }^{c}$ & 1449 & $3.92 \pm 0.02$ & $1.87 \pm 0.05$ & $3.08 \pm 0.23$ & $1.15 \pm 0.13$ \\
\hline$\alpha$-Patchoulene ${ }^{d}$ & 1453 & - & - & $0.47 \pm 0.00$ & $1.85 \pm 0.11$ \\
\hline allo-Aromadedrene $^{d}$ & 1458 & - & - & $2.24 \pm 0.10$ & $4.93 \pm 0.15$ \\
\hline$(E)$-Methyl isoeugenol ${ }^{\mathrm{d}}$ & 1459 & - & - & - & - \\
\hline Dehydro-aromadedrene $e^{d}$ & 1459 & - & - & $3.28 \pm 0.07$ & $4.43 \pm 0.13$ \\
\hline 9-epi-(E)-Caryophyllene ${ }^{d}$ & 1464 & - & - & $1.70 \pm 0.01$ & $3.08 \pm 0.22$ \\
\hline$\gamma$-Gurjunene $e^{d}$ & 1472 & - & - & $1.10 \pm 0.05$ & $4.27 \pm 0.17$ \\
\hline b-Chamigrene ${ }^{d}$ & 1474 & - & - & $0.72 \pm 0.01$ & $2.44 \pm 0.13$ \\
\hline$\gamma$-Muurolene ${ }^{d}$ & 1477 & - & - & - & - \\
\hline$\gamma$-Curcumene ${ }^{d}$ & 1478 & - & - & $0.84 \pm 0.01$ & $1.76 \pm 0.06$ \\
\hline$\gamma$-Himachalene ${ }^{d}$ & 1480 & - & - & - & $2.84 \pm 0.02$ \\
\hline Germacrene $D^{c}$ & 1484 & - & - & $1.94 \pm 0.08$ & $3.56 \pm 0.11$ \\
\hline b-Selinene ${ }^{d}$ & 1486 & - & - & $1.35 \pm 0.10$ & - \\
\hline$\delta$-Selinene ${ }^{d}$ & 1491 & - & - & $2.98 \pm 0.16$ & $4.56 \pm 0.13$ \\
\hline Viridiflorene $^{d}$ & 1494 & - & - & - & $1.88 \pm 0.16$ \\
\hline$(E)-$ Methyl isoeugenol $^{d}$ & 1499 & - & - & - & - \\
\hline Bicyclogermacrene $^{d}$ & 1500 & $6.59 \pm 0.00$ & $2.43 \pm 0.07$ & - & - \\
\hline B-Bisabolene ${ }^{d}$ & 1503 & $0.93 \pm 0.02$ & $1.68 \pm 0.06$ & - & - \\
\hline$\alpha$-Bulnesene ${ }^{d}$ & 1506 & - & - & - & - \\
\hline$\delta$-Cadinene ${ }^{d}$ & 1519 & - & $1.16 \pm 0.05$ & - & - \\
\hline$\delta$-Cadinene ${ }^{d}$ & 1520 & - & $\frac{-}{1.00-0.00}$ & - & - \\
\hline (Z)-Nerolidold & 1534 & $0.70 \pm 0.00$ & - & - & - \\
\hline$\alpha$-Calacorene ${ }^{d}$ & 1544 & - & - & - & - \\
\hline Elemol $^{\mathrm{d}}$ & 1549 & $0.83 \pm 0.01$ & $2.02 \pm 0.04$ & - & - \\
\hline Elemicin $^{d}$ & 1554 & - & $1.01 \pm 0.08$ & - & - \\
\hline
\end{tabular}




\begin{tabular}{|c|c|c|c|c|c|}
\hline 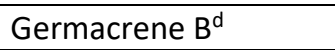 & 1556 & $9.33 \pm 0.26$ & $2.83 \pm 0.07$ & - & - \\
\hline Geranyl butanoate $^{d}$ & 1557 & - & - & - & - \\
\hline b-Calacorene ${ }^{d}$ & 1560 & - & - & - & - \\
\hline Spathulenol ${ }^{\mathrm{c}}$ & 1572 & $16.37 \pm 0.55$ & $1.43 \pm 0.05$ & - & - \\
\hline Caryophyllene oxide $^{c}$ & 1582 & $8.34 \pm 0.22$ & $2.06 \pm 0.01$ & - & - \\
\hline Globulol $^{d}$ & 1589 & - & - & - & - \\
\hline Viridiflorol $^{d}$ & 1590 & - & - & - & - \\
\hline Rosifoliol $^{\mathrm{d}}$ & 1599 & - & - & - & - \\
\hline Guaiol $^{d}$ & 1602 & $4.99 \pm 0.08$ & $18.38 \pm 0.78$ & - & - \\
\hline 6-Himachalene oxide ${ }^{d}$ & 1610 & $0.99 \pm 0.01$ & - & - & - \\
\hline B-Acorenol ${ }^{d}$ & 1631 & - & - & - & - \\
\hline epi- $\alpha$-Muurolol ${ }^{d}$ & 1641 & - & $1.53 \pm 0.07$ & - & - \\
\hline$\alpha$-Muurolol ${ }^{\mathrm{d}}$ & 1645 & - & $0.91 \pm 0.01$ & - & - \\
\hline Cubenol $^{\mathrm{d}}$ & 1645 & - & $2.49 \pm 0.03$ & - & - \\
\hline B-Eudesmol ${ }^{d}$ & 1649 & $1.61 \pm 0.01$ & $2.62 \pm 0.04$ & - & - \\
\hline Himachalol $^{d}$ & 1650 & $1.57 \pm 0.02$ & - & - & - \\
\hline$\alpha$-Cadinol $^{\mathrm{d}}$ & 1654 & - & - & - & - \\
\hline Valerianol $^{d}$ & 1657 & $3.66 \pm 0.11$ & $10.62 \pm 0.67$ & - & - \\
\hline Bulnesol $^{\mathrm{d}}$ & 1666 & $1.42 \pm 0.10$ & $5.84 \pm 0.11$ & - & - \\
\hline Cadalene $^{d}$ & 1670 & & & - & - \\
\hline Foeniculin $^{d}$ & 1677 & $1.99 \pm 0.06$ & $3.26 \pm 0.07$ & - & - \\
\hline Khusinol $^{\mathrm{d}}$ & 1679 & & & - & - \\
\hline$\alpha$-Bisabolol $^{\mathrm{d}}$ & 1681 & - & - & - & - \\
\hline Hexadecanoic acid $^{d}$ & 1955 & - & - & - & - \\
\hline Total & & $93.02 \pm 0.47$ & $98.69 \pm 1.01$ & $97.99 \pm 0.88$ & $97.49 \pm 0.79$ \\
\hline Monoterpenes & & $1.23 \pm 0.04$ & $9.86 \pm 0.11$ & $7.77 \pm 0.10$ & $5.85 \pm 0.05$ \\
\hline Sesquiterpenes & & $91.79 \pm 0.44$ & $87.76 \pm 0.87$ & $90.22 \pm 0.73$ & $91.64 \pm 0.76$ \\
\hline Fatty acid & & - & - & - & - \\
\hline Phenylpropanoids & & - & $1.01 \pm 0.08$ & - & - \\
\hline
\end{tabular}

${ }^{a}$ Retention indices calculated from retention times in relation to those of a series of C8 -C40 n-alkanes on a DB-5 capillary column; bStandard Error; cMethod of identification: Retention Index; Mass Spectroscopy; Co-Injection with authentic compounds; dMethod of identification: Retention Index and Mass Spectroscop

Table No. 1B

Percentage compositions of the essential oils from Croton species

\begin{tabular}{|c|c|c|c|c|c|}
\hline \multirow{3}{*}{ Compound } & \multirow{2}{*}{$\mathbf{R I}^{\mathrm{a}}$} & \multicolumn{2}{|c|}{$\begin{array}{c}\text { C. blanchetianus } \\
\left(\% \pm S E^{b}\right)\end{array}$} & \multicolumn{2}{|c|}{$\begin{array}{l}\text { C. grewioides } \\
\left(\% \pm S E^{b}\right)\end{array}$} \\
\hline & & Leaves & Stems & Leaves & Stems \\
\hline & Yield \% & $0.50 \pm 0.03$ & $0.03 \pm 0.001$ & $2.40 \pm 0.02$ & $0.10 \pm 0.01$ \\
\hline$\alpha$-Pinene ${ }^{c}$ & 929 & - & - & - & - \\
\hline 6-Pinene ${ }^{c}$ & 973 & - & - & - & - \\
\hline$\delta$-3-Carene ${ }^{d}$ & 1005 & - & $0.57 \pm 0.01$ & - & - \\
\hline$o$-Cymene ${ }^{d}$ & 1022 & & & - & - \\
\hline Limonene $^{c}$ & 1021 & - & - & - & - \\
\hline 1,8 -Cineole ${ }^{c}$ & 1031 & & & $1.11 \pm 0.11$ & $0.50 \pm 0.03$ \\
\hline Linalool $^{c}$ & 1092 & - & - & $0.24 \pm 0.02$ & $0.21 \pm 0.00$ \\
\hline trans-Sabinene hydrate ${ }^{d}$ & 1098 & - & - & - & - \\
\hline Myrcenol $^{d}$ & 1113 & - & - & - & - \\
\hline Camphor ${ }^{\mathrm{c}}$ & 1144 & - & - & $0.83 \pm 0.05$ & $1.54 \pm 0.01$ \\
\hline Isoborneol $^{\mathrm{d}}$ & 1156 & - & - & - & - \\
\hline$\alpha$-Terpineol ${ }^{d}$ & 1192 & - & - & $0.53 \pm 0.02$ & $0.92 \pm 0.03$ \\
\hline
\end{tabular}

Boletín Latinoamericano y del Caribe de Plantas Medicinales y Aromáticas/249 


\begin{tabular}{|c|c|c|c|c|c|}
\hline Methyl chavicold & 1196 & - & - & $1.92 \pm 0.11$ & $0.50 \pm 0.00$ \\
\hline$p$-Anisaldehyde ${ }^{d}$ & 1250 & - & - & $0.51 \pm 0.00$ & $1.44 \pm 0.10$ \\
\hline (Z)-Anethole ${ }^{d}$ & 1251 & - & - & $4.60 \pm 0.07$ & $0.82 \pm 0.01$ \\
\hline$(E)$-Anethole ${ }^{d}$ & 1280 & - & - & $55.51 \pm 0.40$ & $37.80 \pm 0.38$ \\
\hline Bornyl acetate $^{d}$ & 1285 & - & - & - & - \\
\hline$\delta$-Elemene ${ }^{d}$ & 1330 & $1.25 \pm 0.01$ & - & - & - \\
\hline$\alpha$-Cubebene $^{d}$ & 1341 & $0.11 \pm 0.00$ & - & - & - \\
\hline$\alpha$-Copaene $^{\mathrm{d}}$ & 1369 & $3.73 \pm 0.11$ & $1.35 \pm 0.05$ & $2.14 \pm 0.12$ & $0.22 \pm 0.00$ \\
\hline 3,4-Dihydro-coumarin ${ }^{d}$ & 1376 & - & - & - & - \\
\hline (Z)-6-Damascenone ${ }^{d}$ & 1381 & $0.37 \pm 0.01$ & - & - & - \\
\hline B-Cubebene ${ }^{d}$ & 1383 & $0.06 \pm 0.00$ & - & - & - \\
\hline B-Bourbonene ${ }^{d}$ & 1384 & $0.13 \pm 0.00$ & - & - & - \\
\hline iso-Longifolene $^{\mathrm{d}}$ & 1385 & - & - & - & - \\
\hline b-Elemene ${ }^{d}$ & 1389 & $0.35 \pm 0.01$ & - & $1.00 \pm 0.04$ & $0.32 \pm 0.00$ \\
\hline Cyperene $^{d}$ & 1398 & - & - & - & - \\
\hline Methyl eugenol $^{\mathrm{C}}$ & 1401 & - & - & $10.63 \pm 0.34$ & $6.61 \pm 0.50$ \\
\hline B-Caryophyllene ${ }^{c}$ & 1414 & $0.81 \pm 0.03$ & - & $4.50 \pm 0.04$ & $0.20 \pm 0.01$ \\
\hline b-Cedrene ${ }^{d}$ & 1416 & - & - & - & - \\
\hline B-Ylangene ${ }^{\mathrm{d}}$ & 1417 & - & - & - & - \\
\hline 6-Duprezianene ${ }^{d}$ & 1418 & - & - & - & - \\
\hline Lavandulyl isobutanoate $^{d}$ & 1419 & - & - & - & - \\
\hline Linalol butanoate $^{d}$ & 1421 & - & - & - & - \\
\hline Dictamnol $^{\mathrm{d}}$ & 1428 & - & - & - & - \\
\hline cis-Thujupsene $^{d}$ & 1429 & - & - & - & - \\
\hline B-Copaene ${ }^{d}$ & 1429 & - & - & - & - \\
\hline B-Gurjunene ${ }^{d}$ & 1430 & $0.12 \pm 0.01$ & - & - & - \\
\hline trans- $\alpha$-Bergamotene $^{d}$ & 1430 & - & - & $0.34 \pm 0.03$ & $0.64 \pm 0.01$ \\
\hline$\gamma$-Elemene ${ }^{\mathrm{d}}$ & 1432 & $0.09 \pm 0.00$ & - & - & - \\
\hline b-Dihydro-ionone ${ }^{d}$ & 1434 & - & - & - & - \\
\hline$\alpha$-Guaiene ${ }^{\mathrm{d}}$ & 1436 & $0.14 \pm 0.00$ & - & - & - \\
\hline Aromadendrene $^{c}$ & 1436 & - & - & - & - \\
\hline$\alpha$-Himachalene ${ }^{\mathrm{d}}$ & 1446 & $1.73 \pm 0.08$ & - & - & - \\
\hline neo- $\alpha$-Clovene ${ }^{d}$ & 1448 & $0.26 \pm 0.03$ & - & - & - \\
\hline$\alpha$-Humulene ${ }^{c}$ & 1449 & $0.29 \pm 0.02$ & - & - & - \\
\hline$\alpha$-Patchoulene ${ }^{d}$ & 1453 & - & - & - & - \\
\hline allo-Aromadedrene $^{\mathrm{d}}$ & 1458 & - & $2.03 \pm 0.10$ & - & - \\
\hline$(E)$-Methyl isoeugenol ${ }^{\mathrm{d}}$ & 1459 & - & - & $2.90 \pm 0.07$ & $0.44 \pm 0.00$ \\
\hline Dehydro-aromadedrene ${ }^{d}$ & 1459 & $0.23 \pm 0.01$ & $1.37 \pm 0.09$ & - & - \\
\hline 9-epi-(E)-Caryophyllene ${ }^{d}$ & 1464 & - & - & - & - \\
\hline$y$-Gurjunene ${ }^{d}$ & 1472 & $0.81 \pm 0.06$ & - & - & - \\
\hline b-Chamigrene ${ }^{d}$ & 1474 & - & - & - & - \\
\hline$\gamma$-Muurolene ${ }^{d}$ & 1477 & $0.69 \pm 0.04$ & - & - & - \\
\hline$y$-Curcumene ${ }^{d}$ & 1478 & - & - & - & - \\
\hline$\gamma$-Himachalene ${ }^{d}$ & 1480 & - & - & - & - \\
\hline Germacrene $D^{c}$ & 1484 & & - & $0.41 \pm 0.00$ & $0.15 \pm 0.02$ \\
\hline b-Selinene ${ }^{d}$ & 1486 & - & - & - & - \\
\hline$\delta$-Selinene ${ }^{d}$ & 1491 & - & - & - & - \\
\hline Viridiflorene $^{d}$ & 1494 & - & - & - & - \\
\hline$(E)-$ Methyl isoeugenol ${ }^{\mathrm{d}}$ & 1499 & - & - & $6.70 \pm 0.10$ & $31.03 \pm 0.33$ \\
\hline Bicyclogermacrene $^{d}$ & 1500 & - & - & - & - \\
\hline B-Bisabolene ${ }^{d}$ & 1503 & - & - & - & - \\
\hline$\alpha$-Bulnesene ${ }^{d}$ & 1506 & - & $2.65 \pm 0.05$ & - & - \\
\hline
\end{tabular}




\begin{tabular}{|c|c|c|c|c|c|}
\hline$\delta$-Cadinene ${ }^{\mathrm{d}}$ & 1519 & $17.86 \pm 0.33$ & $6.80 \pm 0.20$ & - & - \\
\hline$\delta$-Cadinene ${ }^{d}$ & 1520 & - & - & $1.32 \pm 0.04$ & $0.21 \pm 0.00$ \\
\hline (Z)-Nerolidol ${ }^{\mathrm{d}}$ & 1534 & - & - & - & - \\
\hline$\alpha$-Calacorene ${ }^{d}$ & 1544 & $2.49 \pm 0.10$ & $1.77 \pm 0.16$ & - & - \\
\hline Elemol $^{d}$ & 1549 & - & - & - & - \\
\hline Elemicin $^{d}$ & 1554 & - & - & - & - \\
\hline Germacrene $B^{d}$ & 1556 & - & - & - & - \\
\hline Geranyl butanoate $^{d}$ & 1557 & $1.09 \pm 0.01$ & - & - & - \\
\hline b-Calacorene ${ }^{\mathrm{d}}$ & 1560 & $0.89 \pm 0.02$ & - & - & - \\
\hline Spathulenolc & 1572 & $24.1 \pm 0.28$ & $43.52 \pm 1.01$ & $1.63 \pm 0.01$ & $0.91 \pm 0.02$ \\
\hline Caryophyllene oxide $^{c}$ & 1582 & - & - & $2.84 \pm 0.05$ & $2.53 \pm 0.10$ \\
\hline Globulol $^{d}$ & 1589 & $7.30 \pm 0.15$ & $6.06 \pm 0.32$ & - & - \\
\hline Viridiflorol $^{d}$ & 1590 & $3.61 \pm 0.09$ & - & - & - \\
\hline Rosifoliol $^{d}$ & 1599 & - & $3.01 \pm 0.16$ & - & - \\
\hline Guaiol $^{\mathrm{d}}$ & 1602 & - & - & - & - \\
\hline 6-Himachalene oxide ${ }^{d}$ & 1610 & - & - & - & - \\
\hline b-Acorenol ${ }^{d}$ & 1631 & $11.16 \pm 0.09$ & $13.38 \pm 0.21$ & - & $2.62 \pm 0.11$ \\
\hline epi- $\alpha$-Muurolol ${ }^{d}$ & 1641 & - & - & - & - \\
\hline$\alpha$-Muurolol ${ }^{d}$ & 1645 & - & - & - & - \\
\hline Cubenol $^{\mathrm{d}}$ & 1645 & $2.02 \pm 0.06$ & $2.59 \pm 0.06$ & $0.51 \pm 0.00$ & $1.01 \pm 0.12$ \\
\hline 6-Eudesmol ${ }^{d}$ & 1649 & - & - & - & - \\
\hline Himachalol $^{d}$ & 1650 & - & - & - & - \\
\hline$\alpha$-Cadinol $^{\mathrm{d}}$ & 1654 & $6.73 \pm 0.11$ & $7.38 \pm 0.19$ & - & - \\
\hline Valerianol $^{d}$ & 1657 & - & - & - & - \\
\hline Bulnesol $^{d}$ & 1666 & - & - & - & - \\
\hline Cadalene $^{d}$ & 1670 & - & $3.13 \pm 0.21$ & - & $8.42 \pm 0.08$ \\
\hline Foeniculin $^{d}$ & 1677 & - & - & - & - \\
\hline Khusinol $^{\mathrm{d}}$ & 1679 & $1.21 \pm 0.02$ & $2.89 \pm 0.07$ & - & - \\
\hline$\alpha$-Bisabolol $^{\mathrm{d}}$ & 1681 & $0.93 \pm 0.03$ & - & - & - \\
\hline Hexadecanoic acid ${ }^{d}$ & 1955 & $9.02 \pm 0.18$ & - & - & - \\
\hline Total & & $99.58 \pm 0.97$ & $98.50 \pm 0.80$ & $98.80 \pm 0.51$ & $98.64 \pm 0.57$ \\
\hline Monoterpenes & & - & $0.57 \pm 0.01$ & $9.64 \pm 0.11$ & $5.82 \pm 0.10$ \\
\hline Sesquiterpenes & & $90.56 \pm 0.93$ & $97.93 \pm 0.78$ & $14.54 \pm 0.05$ & $6.13 \pm 0.13$ \\
\hline Fatty acid & & $9.02 \pm 0.18$ & - & - & - \\
\hline Phenylpropanoids & & - & - & $75.7 \pm 0.48$ & $75.8 \pm 0.41$ \\
\hline
\end{tabular}

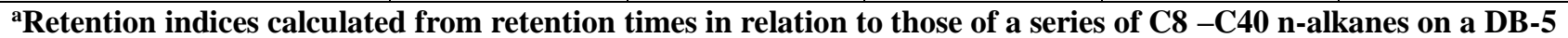
capillary column; bStandard Error; cMethod of identification: Retention Index; Mass Spectroscopy; Co-Injection with authentic compounds; dMethod of identification: Retention Index and Mass Spectroscop

Twenty-eight compounds were identified in the $C$. heliotropiifolius oils, accounting for $93.02 \pm$ $0.47 \%$ and $98.69 \pm 1.01 \%$ of the leaf and stem oils, respectively. $\beta$-Caryophyllene $(20.82 \pm 0.38 \%)$ and spathulenol $(16.37 \pm 0.55 \%)$ were identified as the major constituents of the leaf oil, whereas guaiol $(18.38 \pm 0.78 \%), \beta$-elemene $(17.28 \pm 079 \%)$ and valerianol $(10.62 \pm 0.67 \%)$ were the major constituents of the stem oil.

Thirty-seven compounds were identified in the $C$. adenocalyx oils, accounting for $97.99 \pm 0.88 \%$ and $97.49 \pm 0.79 \%$ of the leaf and stem oils, respectively. The analysis revealed a highly similar chemical composition between the two oils, with $\beta$ caryophyllene $(15.64 \pm 0.62 \% / 12.21 \pm 0.72 \%), \gamma$ elemene $(14.80 \pm 0.84 \% / 6.85 \pm 0.12 \%)$ and $\beta$ copaene $(11.45 \pm 0.32 \% / 8.45 \pm 0.28 \%)$ as the major constituents of the leaf/stem oil.

Thirty-six compounds were identified in the C. blanchetianus oils, accounting for $99.58 \pm 0.97 \%$ and $98.50 \pm 0.80 \%$ of the leaf and stem oils, respectively. Spathulenol $(24.1 \pm 0.28 \%$ / $43.52 \pm$ $1.01 \%), \delta$-cadinene $(17.86 \pm 0.33 \% / 6.80 \pm 0.20 \%)$ and $\beta$-acorenol $(11.16 \pm 0.09 \% / 13.38 \pm 0.21 \%)$ 
were the major constituents of the leaf/stem oil. A significant percentage of hexadecanoic acid (9.02 \pm $0.18 \%$ ) was also found in the leaf oil.

Twenty-two compounds were found in the $C$. grewioides oils, accounting for $98.80 \pm 0.51 \%$ and $98.64 \pm 0.57 \%$ of the leaf and stem oils, respectively. Phenopropanoids constituted the predominant chemical class, corresponding to $75.7 \pm 0.48 \%$ and $75.8 \pm 0.41 \%$ of the leaf and stem oils, respectively. The two oils had a similar chemical profile. With the exception of $\beta$-acorenol $(2.62 \pm 0.11 \%)$ and cadalene $(8.42 \pm 0.08 \%)$, the other compounds were found in the oils from both parts of the plants. The major constituent of the leaf oil was (E)-anethole (55.51 \pm $0.40 \%$ ), followed by methyl eugenol (10.63 \pm $0.34 \%)$. The major constituent of the stem oil was (E)-anethole $(37.80 \pm 0.38 \%)$, followed by (E)methyl isoeugenol $(31.03 \pm 0.33 \%)$.

Table No. 2 displays the mean lethal $\left(\mathrm{LC}_{50}\right)$ and repellency $\left(\mathrm{RC}_{50}\right)$ concentrations for the essential oils from the leaves and stems of the four Croton species. All oils were toxic to T. urticae and exhibited repellent properties against the mite.

Table No. 2

Toxicity by residual contact and repellence activity of essential oil of four species Croton collected in Pernambuco-Brazil against Tetranychus urticae

\begin{tabular}{|c|c|c|c|c|c|c|c|}
\hline Essential oil & & Bioassay & $\mathbf{N}^{\mathbf{a}}$ & $\mathbf{D F}^{\mathbf{b}}$ & Slope $\pm \mathbf{S E}^{\mathbf{c}}$ & $\operatorname{Activity}^{\mathrm{d}}\left(\mathrm{CI}^{\mathrm{e}} 95 \%\right)$ & $\chi^{2 \mathrm{f}}$ \\
\hline \multirow{4}{*}{ C. heliotropiifolius } & \multirow{2}{*}{ leave } & Contact & 720 & 6 & $2.37 \pm 0.14$ & $46.83(41.84-52.25)$ & 5.08 \\
\hline & & Repellence & 1200 & 6 & $1.46 \pm 0.10$ & $9.26(7.501-11.06)$ & 10.53 \\
\hline & \multirow{2}{*}{ stems } & Contact & 720 & 6 & $2.35 \pm 0.14$ & $52.63(47.07-58.67)$ & 4.29 \\
\hline & & Repellence & 1200 & 6 & $2.15 \pm 0.13$ & $27.08(23.97-30.13)$ & 6.42 \\
\hline \multirow{4}{*}{ C. adenocalyx } & \multirow{2}{*}{ leaves } & Contact & 720 & 6 & $0.70 \pm 0.04$ & $0.97(0.61-1.44)$ & 8.84 \\
\hline & & Repellence & 1200 & 6 & $0.70 \pm 0.04$ & $0.11(0.08-0.16)$ & 8.79 \\
\hline & \multirow{2}{*}{ stems } & Contact & 630 & 5 & $0.88 \pm 0.05$ & $2.36(1.63-3.26)$ & 4.81 \\
\hline & & Repellence & 1200 & 6 & $0.71 \pm 0.04$ & $0.14(0.09-0.18)$ & 9.96 \\
\hline \multirow{4}{*}{ C. blanchetianus } & \multirow{2}{*}{ leaves } & Contact & 720 & 6 & $1.88 \pm 0.11$ & $2.95(2.58-3.38)$ & 4.52 \\
\hline & & Repellence & 1200 & 6 & $0.71 \pm 0.03$ & $0.19(0.14-0.25)$ & 7.34 \\
\hline & & Contact & 720 & 6 & $2.14 \pm 0.13$ & $17.77(15.67-20.07)$ & 6.38 \\
\hline & stems & Repellence & 1200 & 6 & $0.90 \pm 0.05$ & $1.67(1.31-2.09)$ & 8.71 \\
\hline \multirow{4}{*}{ C. grewioides } & \multirow{2}{*}{ leaves } & Contact & 720 & 6 & $1.42 \pm 0.10$ & $21.01(16.68-25.66)$ & 6.23 \\
\hline & & Repellence & 1200 & 6 & $1.12 \pm 0.06$ & 7.05 (5.77-8.48) & 6.47 \\
\hline & & Contact & 720 & 6 & $1.37 \pm 0.10$ & $28.64(22.80-34.89)$ & 8.24 \\
\hline & stems & Repellence & 1200 & 6 & $1.06 \pm 0.06$ & $6.70(5.39-8.15)$ & 9.44 \\
\hline \multirow{2}{*}{$\operatorname{Azamax}^{\circledR}$} & & Contact & 630 & 5 & $2.46 \pm 0.19$ & $0.31(0.28-0.36)$ & 8.42 \\
\hline & & Repellence & 1050 & 5 & $0.97 \pm 0.09$ & $0.04(0.03-0.21)$ & 1.02 \\
\hline Ortus & & Contact & 810 & 7 & $1.04 \pm 0.07$ & $0.15(0.08-0.25)$ & 8.66 \\
\hline
\end{tabular}

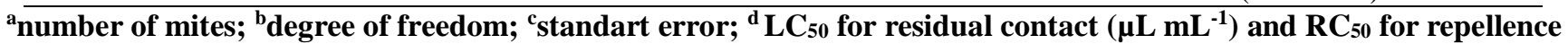
activity $\left(\mu \mathrm{L} \mathrm{mL}{ }^{-1}\right)$; ${ }^{\mathrm{e}}$ confidence interval; ${ }^{\mathrm{f}}$ chi-square.

The susceptibility of the mite and the repellent action of the oils varied according to the species and part of the plant studied. The toxicity of the leaf and stem oils from the same species only differed for C. adenocalyx and C. blanchetianus and the repellent action of the leaf and stem oils from the same species only differed for C. heliotropiifolius and $C$. blanchetianus. The oils with the greatest toxicity toward the mite were those from $C$. adenocalyx, from which the leaf and stem oils were threefold and 7.5-fold more toxic than the oils from C. blanchetianus, 21.7-fold and 12-fold more toxic than the oils from $C$. grewioides as well as 48.3 -fold and 22.3-fold more toxic than the oils from $C$. heliotropiifolius, respectively.

In the repellency assays, two groups with different levels were found for the leaf oils. Group 1 was formed by the $C$. adenocalyx and blanchetianus oils and was approximately nine-fold more repellent than Group 2, which was composed of the $C$. grewioides and $C$. heliotropiifolius oils. The following was the order of repellency for the stem oils: $C$. adenocalyx $>C$. blanchetianus $>C$. grewioides $>C$. heliotropiifolius 


\section{Monoterpenes}

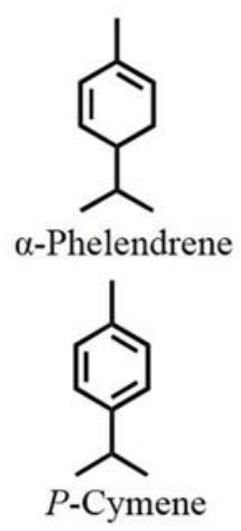<smiles>C=C1C=CC(C(C)C)CC1</smiles>

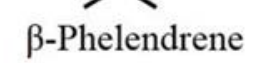<smiles>CC12CCC(C)(CC1)O2</smiles>

1,8-Cineole

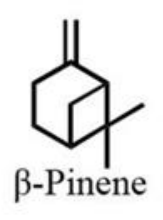<smiles>CC1=CCC2(C1)C(C)C1CCCC12C</smiles><smiles>CC1(C)C2CCC1C(=O)C2</smiles>

Camphor

\section{Phenylpropanoids}<smiles>C=CCc1ccc(OC(C)=O)c(OC(C)(C)CC)c1</smiles>

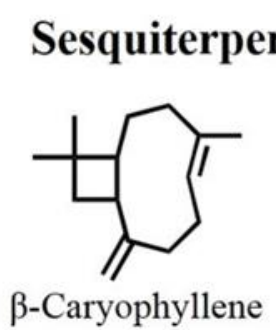

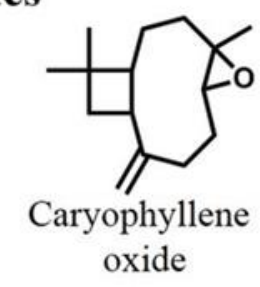<smiles>CC1(O)CCC2C(=C=[As])CCC3C(C21)C3(C)C</smiles><smiles></smiles>

Bicyclogermacrene

Figure No. 1 Structures of the main compounds found in Croton essential oils

In the comparison with the synthetic and plant-based commercial acaricides used as positive controls, none of the oils investigated was more toxic to T. urticae than Ortus ${ }^{\circledR}$ and Azamax ${ }^{\circledR}$. Regarding the repellent action, the $C$. adenocalyx (leaf and stem) and $C$. blanchetianus (leaf) oils had the same level of repellency as Azamax ${ }^{\circledR}$.

To determine the relative lethal and sublethal properties of the major chemical constituents found in the Croton oils, seven monoterpenes, four phenylpropanoids and four sesquiterpenes were selected for the investigation of toxicity by residual contact and repellent action against $T$. urticae. Figure No. 1 presents the chemical structures of the selected constituents classified according to their biogenetic origins. 
Table No. 3

Toxicity by residual contact and repellence of the selected constituents against Tetranychus urticae.

\begin{tabular}{|c|c|c|c|c|c|c|}
\hline Constituents & Bioassay & $\mathbf{N}^{\mathbf{a}}$ & $\mathbf{D F}^{\mathbf{b}}$ & Slope $\pm \mathbf{S E}^{c}$ & $\begin{array}{c}\text { Activity }^{\mathrm{d}} \\
\left(\text { CI }^{\mathrm{e}} \text { 95\%) }\right.\end{array}$ & $\chi^{2 \mathrm{f}}$ \\
\hline \multirow{2}{*}{ eugenol } & Contact & 630 & 5 & $2.01 \pm 0.34$ & $9.13(7.62-10.12)$ & 7.39 \\
\hline & Repellency & 240 & 6 & $0.35 \pm 0.02$ & $0.001(0.0009-0.002)$ & 2.19 \\
\hline \multirow{2}{*}{$\alpha$-pinene } & Contact & 810 & 7 & $1.55 \pm 0.11$ & $26.77(21.81-33.49)$ & 7.49 \\
\hline & Repellency & 240 & 6 & $1.78 \pm 0.27$ & $2.37(1.63-3.06)$ & 7.28 \\
\hline \multirow{2}{*}{$\beta$-pinene } & Contact & 630 & 5 & $1.45 \pm 0.09$ & $27.80(23.38-36.13)$ & 1.98 \\
\hline & Repellency & 240 & 6 & $3.67 \pm 0.41$ & $3.04(2.67-3.43)$ & 2.56 \\
\hline \multirow{2}{*}{ 1.8-cineole } & Contact & 630 & 5 & $1.62 \pm 0.20$ & $81.80(72.79-107.84)$ & 4.98 \\
\hline & Repellency & 210 & 5 & $6.30 \pm 0.73$ & $6.07(5.52-6.57)$ & 1.42 \\
\hline \multirow{2}{*}{ camphor } & Contact & 720 & 6 & $2.02 \pm 0.13$ & $10.29(8.91-11.74)$ & 8.90 \\
\hline & Repellency & 240 & 6 & $4.50 \pm 0.52$ & $5.01(4.49-5.52)$ & 10.19 \\
\hline \multirow{2}{*}{ (E)-anethole } & Contact & 720 & 6 & $1.23 \pm 0.07$ & $1.49(1.19-1.84)$ & 9.98 \\
\hline & Repellency & 210 & 5 & $1.25 \pm 0.15$ & $0.02(0.01-0.03)$ & 1.45 \\
\hline \multirow{2}{*}{ methyl eugenol } & Contact & 720 & 6 & $2.04 \pm 0.15$ & $7.67(6.82-8.61)$ & 4.00 \\
\hline & Repellency & 210 & 5 & $1.21 \pm 0.14$ & $0.001(0.0007-0.002)$ & 5.34 \\
\hline \multirow{2}{*}{$\beta$-caryophyllene } & Contact & 630 & 5 & $2.25 \pm 0.28$ & $0.74(0.56-0.91)$ & 1.27 \\
\hline & Repellency & 240 & 6 & $2.17 \pm 0.23$ & $5.07(4.08-6.21)$ & 1.15 \\
\hline \multirow{2}{*}{ bicyclogermacrene } & Contact & 720 & 6 & $1.48 \pm 0.08$ & $4.95(4.14-5.88)$ & 9.87 \\
\hline & Repellency & 240 & 6 & $2.25 \pm 0.24$ & $18.28(14.80-22.19)$ & 4.23 \\
\hline \multirow{2}{*}{ spathulenol } & Contact & 630 & 5 & $1.09 \pm 0.06$ & $0.70(0.54-0.90)$ & 8.23 \\
\hline & Repellency & 240 & 6 & $2.38 \pm 0.25$ & $9.51(7.79-11.43)$ & 2.80 \\
\hline \multirow{2}{*}{ caryophyllene oxide } & Contact & 630 & 5 & $1.97 \pm 0.20$ & $105.58(78.82-125.12)$ & 8.02 \\
\hline & Repellency & 240 & 6 & $2.54 \pm 0.27$ & $13.77(11.50-16.35)$ & 3.81 \\
\hline \multirow{2}{*}{$\rho$-cymene } & Contact & 810 & 7 & $1.45 \pm 0.21$ & $28.53(22.35-35.45)$ & 9.38 \\
\hline & Repellency & 240 & 6 & $1.92 \pm 0.20$ & $0.90(0.71-1.13)$ & 3.86 \\
\hline \multirow{2}{*}{ eugenyl acetate } & Contact & 810 & 7 & $1.90 \pm 0.11$ & $5.67(5.01-6.45)$ & 0.27 \\
\hline & Repellency & 210 & 5 & $1.34 \pm 0.16$ & $0.002(0.001-0.004)$ & 7.87 \\
\hline \multirow{2}{*}{$\alpha$-phellandrene } & Contact & 720 & 6 & $2.76 \pm 0.19$ & $40.80(37.21-44.69)$ & 3.66 \\
\hline & Repellency & 240 & 6 & $1.67 \pm 0.26$ & $2.57(1.78-3.34)$ & 7.08 \\
\hline \multirow{2}{*}{$\beta$-phellandrene } & Contact & 720 & 6 & $2.07 \pm 0.13$ & $2.67(2.36-3.02)$ & 5.91 \\
\hline & Repellency & 210 & 5 & $2.12 \pm 0.24$ & $2.13(1.27-2.73)$ & 3.22 \\
\hline
\end{tabular}

${ }^{a}$ number of mites; ${ }^{b}$ degree of freedom; ' standart error; ${ }^{d} L_{50}$ for residual contact $\left(\mu \mathrm{L} \mathrm{mL}^{-1}\right)$ and $\mathrm{RC}_{50}$ for repellence activity $\left(\mu \mathrm{L} \mathrm{mL}{ }^{-1}\right)$; ${ }^{\mathrm{e}}$ confidence interval; ${ }^{\mathrm{f}}$ chi-square.

Based on the $\mathrm{LC}_{50}$ (toxicity) and $\mathrm{RC}_{50}$ (repellent effect) values estimated for the selected constituents (Table No. 3), all compounds were toxic by residual contact and acted as repellents to the mite.

Toxicity by residual contact varied according to the chemical class of the compounds. Although the results indicated an increasing order of toxicity (monoterpenes < phenylpropanoids < sesquiterpenes), it was not possible to establish this order for all compounds investigated. For example, while the sesquiterpenes $\beta$-caryophyllene and spatulenol (with the same level of toxicity) were more toxic to the mite, the sesquiterpene caryophyllene oxide was the least toxic of all the constituents tested. Among the phenylpropanoids, the greatest toxicity was found for $(E)$-anethole, followed by eugenol, eugenyl acetate and methyl eugenol (with the same level of toxicity). Regarding the monoterpenes, $\beta$-phellandrene exhibited the greatest toxicity, followed by camphor.

In terms of repellency according to the chemical class of the compounds investigated, phenylpropanoids were the most repellent, followed by monoterpenes and sesquiterpenes. The exception was the sesquiterpene $\beta$-caryophyllene, which had the same level of repellent action as the oxygenated monoterpenes 1,8-cienol and camphor. Among the 
phenylpropanoids, eugenol, methyl eugenol and eugenyl acetate (with the same level of repellency) were the most repellent. Non-oxygenated monoterpenes were more repellent that the oxygenated forms, particularly $\alpha$ and $\beta$-phellandrene as well as $\alpha$ and $\beta$-pinene, which had the same level of repellency. Among the sesquiterpenes tested, bicyclogermacrene and caryophyllene oxide had the lowest repellent action (Table No. 3).

\section{DISCUSSION}

The $C$. grewioides oils were rich in phenylpropanoids, whereas sesquiterpenes were the predominant chemical class in the $C$. heliotropiifolius, $C$. adenocalyx and $C$. blanchetianus oils. These results are in agreement with data reported for other species of the genus (Dória et al., 2010; Filho et al., 2017; Castro et al., 2019).

The major chemical constituents of the Croton species investigated herein have also been reported for these species occurring in other locations of northeast Brazil. For instance, $\beta$-caryophyllene, which was identified as the major constituent of the C. adenocalyx and C. heliotropiifolius oils in the present investigation, was also reported as the major constituent of the leaf oil from $C$. heliotropiifolius (28.61 to $46.99 \%)$ collected in the state of Pernambuco (Filho et al., 2017) as well as $C$. blanchetianus (14.58\%) and C. adenocalyx (10.23\%) collected in the state of Ceará (Pinho-da-Silva et al., 2010; De Lima et al., 2010). For C. heliotropiifolius, $\beta$-caryophyllene was also found as the major constituent in the oils from the shoots of samples collected in the states of Bahia $(23.85 \pm 0.36 \%)$ (Araújo et al., 2017) and Sergipe (35.82\%) (Dória et al., 2010).

$\beta$-Elemene, which was the major constituent of the stem oil from $C$. heliotropiifolius, was also reported for the leaf oil from C. adenocalyx $(8.31 \%)$ occurring in the state of Ceará (De Lima et al., 2010). For the $C$. blanchetianus oils, we found the same chemotype [spathulenol] (leaf oil $=24.1 \pm 0.28 \%$; stem oil $=43.52 \pm 1.01 \%$ ) described for samples of the species collected in the states of Ceara $(38.32 \%)$ (Lima et al., 2013) and Pernambuco (31.5-10.3\%) (Souza et al., 2017). The chemical profile found in the present study for the leaf and stem oils from $C$. grewioides, with (E)-anethole as the major constituent, is in agreement with data reported by Silva et al. (2008) for the same species collected in the state of Pernambuco. On the other hand, Castro et al. (2019) investigated two samples collected in the state of Piauí and found the phenylpropanoid methyl chavicol as the major constituent $(83.59 \%$ in São João do Piaui and $95.38 \%$ in Caxingó), which is the isomer of $(E)$-anethole.

As expected, qualitative and quantitative differences were found in the chemical profiles of the essential oils among the species of Croton. Another finding of the present study was the similarity in the chemical profile of the oils from the species $C$. heliotropiifolius, $C$. adenocalyx and $C$. blanchetianus to profiles described in previous studies involving these species collected from different locations and in different seasons. This is an important finding for the standardization of a chemical profile that can serve as the basis for the formulation of an acaricide for use in the management of $T$. urticae by family farmers in agricultural niches in the state of Pernambuco.

Plants and their extracts have been used for millennia to protect against or repel arthropods. There are several reports in the literature in which essential oils from different taxa, basically comprising monoterpenes, sesquiterpenes and phenylpropanoids, have consistently shown activity against a variety of arthropods (Nerio et al., 2010; Mossa, 2016). Evidence regarding the obtainment of a product formulated on the basis of essential oils for the control of mites and/or insects can be found in the scientific literature, including records of patents with native and cultivated aromatic plants in northeast Brazil (Araújo et al., 2015; Melo \& da Camara, 2019).

In the present study, the differences in relative toxicity and repellent action against $T$. urticae obtained for the essential oils from $C$. heliotropiifolius, C. adenocalyx, C. blanchetianus and $C$. grewioides can be attributed to quantitative and qualitative differences in the chemical composition, as determined by GC-MS.

With the exception of the $C$. grewioides oil, which was evaluated for its effect on the cattle tick Rhipicephalus microplus (Castro et al., 2019), previous investigations of the biological properties of Croton oils were limited to insecticidal action against Nasutitermes corniger (C. blanchetianus), an important structural pest in the Neotropics (Lima et al., 2013), larvae of the mosquito Aedes aegypti, which is the main transmitter of dengue, zika and chikungunya (C. heliotropifolius and $C$. blanchetianus) (Morais et al., 2006; Doria et al., 2010), and the storage grain pest Zabrotes subfasciatus (C. grewioides) (Silva et al., 2008).

A comparison of the acaricidal activity of the C. grewioides oil from Caxingó in the state of Piauí (northeast Brazil) against $R$. microplus larvae and 
adults (Castro et al., 2019) and the activity found in the present study against $T$. urticae adults indicates that the $C$. grewioides oil from the state of Pernambuco, Brazil is much more efficient. This difference may be explained by differences between the arthropods, the chemical composition of the oils and the methods used for the assessment of the acaricidal action.

The present findings reveal that the Croton oils are more efficient against $T$. urticae through residual contact than oils from the leaves of $C$. rhamnifolioides from three localities in the state of Pernambuco, Brazil against the same pest. Indeed, the best result in the present study was obtained with the oil from $C$. adenocalyx, which was 2.5 -fold more toxic than the oil from $C$. rhamnifolioides collected in the municipality of Buíque in the state of Pernambuco (da Camara et al., 2017). These results may be explained by differences in the chemical composition of the oils investigated.

This is the first report of the acaricidal action of Croton oils against T. urticae and the first report of the biological property of the essential oil from $C$. adenocalyx. None of the oils investigated in this study was more toxic to $T$. urticae than the commercial acaricides use as positive controls. However, as complex mixtures of monoterpenes, sesquiterpenes and/or phenylpropanoids, essential oils can affect different target sites at the same time, thereby delaying the development of resistant pest populations in comparison to a single active ingredient in a conventional insecticide (Koul \& Walia, 2009) or even a biopesticide, such as azadirachtin (Feng \& Isman, 1995).

The extensive use of acaricidal agents for the control of T. urticae in agricultural niches in the state of Pernambuco, Brazil has led to the development of populations resistant to the main classes of acaricides currently recommended and used in Brazil, including fenpyroximate, which is the active ingredient of Ortus $^{\circledR} 50$ SC (Sato et al., 2004) used in the present investigation as one of the positive controls.

Toxicity by residual contact of the chemical constituents found in essential oils against $T$. urticae has been investigated, including the same compounds analyzed in the present study, such as $\rho$-cymene, $\beta$ caryophyllene, $\beta$-pinene, $\alpha$-pinene, 1,8 -cineole, camphor, bicyclogermacrene and eugenol (Attia et al., 2012; Araújo et al., 2012; Moraes et al., 2017; de Melo et al., 2018; Hong et al., 2018; Born et al., 2018; Cao et al., 2019; Abdelgaleil et al., 2019;). However, this is the first report of the acaricidal activity against this pest of the constituents $(E)$ - anethole, methyl eugenol, spathulenol, caryophyllene oxide, eugenyl acetate, $\alpha$-phellandrene and $\beta$ phellandrene.

The greater toxicity of $\beta$-pinene compared to $\alpha$-pinene to larvae of $A$. aegypti has been attributed to the influence of the exocyclic double bond (Lucia et al., 2007; Perumalsamy et al., 2009). In the present investigation, these monoterpenes had the same level of toxicity by residual contact and the same repellent action. However, the influence of the exocyclic double bond on toxicity was evident in this study for $\beta$-phellandrene, which was 15.3 -fold more toxic to $T$. urticae than its isomer, $\alpha$-phellandrene. On the other hand, the exocyclic double bond of $\beta$-phellandrene was not a determinant in the repellent activity, as the same level of repellency was found for this compound and $\alpha$-phellandrene.

A previous investigation of the repellent action of the chemical constituents of essential oils demonstrated that phenylpropanoids constitute one of the most efficient chemical classes in terms of repellency to arthropods (Nerio et al., 2010). This statement is supported by the present findings, as phenylpropanoids were more repellent to $T$. urticae than monoterpenes and sesquiterpenes. One noteworthy point in the results obtained for the monoterpenes is that, although García et al. (2005) found that the presence of the hydroxyl group in terpenes increased the repellency to Tribolium castaneum, non-oxygenated monoterpenes were more repellent to $T$. urticae than oxygenated monoterpenes in the present study. These results suggest that it is not possible to generalize the repellent action of a molecule by only considering the functional group; other aspects should be considered, such as the interaction between the molecule and target site, which can vary among pests.

Although we found a relation between the chemical class and repellent action for the compounds selected in the present study, in which phenylpropanoids were more efficient than terpenes, other aspects related to the physical properties of the compounds, such as boiling point and vapor pressure, should be taken into consideration (Paluch et al., 2010).

\section{CONCLUSIONS}

The chemical analysis using GC-MS in the present study enabled the determination of qualitative and quantitative differences among the essential oils of the four species of Croton, independently of the part of the plant from which the oils were extracted. Sesquiterpenes constituted the predominant chemical 
class in the leaf and stem oils from $C$. heliotropiifolius, $C$. adenocalyx and $C$. blanchetianus. In contrast, phenylpropanoids were the predominant chemical class in the leaf and stem oils from C. grewioides.

Based on toxicity by residual contact and repellent action, the Croton oils investigated here, particularly the oils from $C$. adenocalyx, constitute a promising alternative to synthetic acaricides for use in the control of $T$. urticae in family farming activities in the semiarid region of the state of Pernambuco, Brazil. However, prior to their use, further studies are required addressing safety issues for human health and the proper formulation to improve the acaricidal potency.

\section{ACKNOWLEDGEMENTS}

This work was supported by Fundação de Amparo à Ciência e Tecnologia do Estado de Pernambuco (FACEPE) [grant number APQ-1008-1.06/15; APQ0476-1.06/14; APQ-08601.06/16; IBPG-07151.06/18]; Conselho Nacional de Desenvolvimento Científico e Tecnológico (CNPq) [grant number PQ2-302860/2016-9] and Coordenação de Aperfeiçoamento de Pessoal de Nível Superior for awarding a grant (CAPES) [grant number PROCAD88887.308194/2018-00].

\section{REFERENCES}

Abdelgaleil SAM, Badawy MEI, Mahmoud NF, Marei AESM. 2019. Acaricidal activity, biochemical effects and molecular docking of some monoterpenes against two-spotted spider mite (Tetranychus urticae Koch). Pest Biochem Physiol 156: 105 - 115. https://doi.org/10.1016/j.pestbp.2019.02.006

Adams RP. 2017. Identification of essential oil components by gas chromatography-mass spectrometry, 4.1th ed., Allured Publishing Corporation, Carol Stream, Illinois, USA. https://doi.org/10.1016/s1044-0305(97)00026-3

Araújo MJC, Câmara CAG, Born FS, Moraes MM, Badji CA. 2012. Acaricidal activity and repellency of essential oil from Piper aduncum and its components against Tetranychus urticae. Exp Appl Acarol 57: 139 - 155. https://doi.org/10.1007/s10493-012-9545-x

Araújo MJC, Da Camara CAG, Born FS. 2015. Produto a base de uma mistura natural contendo óleos essenciais com efeitos sinérgicos e aditivos para o controle de pragas agrícolas. BR Patent No. 1020150164220. Instituto Nacional da Propriedade Industrial, Brasilia, Brasil.

Araújo FM, Dantas MCSM, Silva LS, Aona LYS, Tavares IF, Souza-Neta LC. 2017. Antibacterial activity and chemical composition of the essential oil of Croton heliotropiifolius Kunth from Amargosa, Bahia, Brazil. Ind Crop Prod 105: 203 - 206. https://doi.org/10.1016/j.indcrop.2017.05.016

Attia S, Grissa KL, Mailleux AC, Heuskin S, Lognay G, Hance T. 2012. Acaricidal activities of Santolina africana and Hertia cheirifolia essential oils against the two-spotted spider mite (Tetranychus urticae). Pest Manag Sci 68: 1069 - 1076. https://doi.org/10.1002/ps.3269

Berry PE, Hipp AL, Wurdack KJ, Van EB, Riina R. 2005. Molecular phylogenetics of the giant genus Croton and tribe Crotoneae (Euphorbiaceae sensu stricto) using ITS and trnL-trnF DNA sequence data. Am J Bot 92 : 1520 - 1534. https://doi.org/10.3732/ajb.92.9.1520

Born FS, da Camara CAG, Melo JPR, Moraes MM. 2018. Acaricidal property of the essential oil from Lippia gracilis against Tetranychus urticae and a natural enemy, Neoseiulus californicus, under greenhouse conditions. Exp Appl Acarol 75: 491 - 502. https://doi.org/10.1007/s10493-018-0286-3

Cao J, Pang X, Guo S, Wang Y, Geng Z, Sang Y, Du S. 2019. Pinene-rich essential oils from Haplophyllum dauricum (L.) G. Don display anti-insect activity on two stored-product insects. Int Biodeterior Biodegradation 140: 1 - 8. https://doi.org/10.1016/j.ibiod.2019.03.007

Castro KNC, Chagas ACS, Costa-Junior LM, Canuto KM, Brito ES, Rodrigues THS, de Andrade IM. 2019. Acaricidal potential of volatile oils from Croton species on Rhipicephalus microplus. Braz J Pharmacog 6: 5 pages. https://doi.org/10.1016/j.bjp.2019.09.001

Cruz RCD, Silva SLCE, Souza IA, Gualberto SA, Carvalho KS, Santos FR, Carvalho MG. 2017. Toxicological evaluation of essential oil from the leaves of Croton argyrophyllus (Euphorbiaceae) on Aedes aegypti (Diptera: Culicidae) and Mus musculus (Rodentia: Muridae). J Med Entomol 54: 985 - 993.

https://doi.org/10.1093/jme/tjw239

da Camara CAG, de Moraes MM, de Melo JPR, da Silva MMC. 2017. Chemical composition and acaricidal activity of essential oils from Croton rhamnifolioides Pax and Hoffm. in different regions of a Caatinga 
Biome in Northeastern Brazil. J Essent Oil Bear Plant 20: 1434 - 1449. https://doi.org/10.1080/0972060x.2017.1416677

de Lima SG, Citó AMGL, Lopes JAD, Neto JMM, Chaves MH, Silveira ER. 2010. Fixed and volatile constituents of genus Croton plants: C. adenocalyx Baill - Euphorbiaceae. Rev Latinoamer Quím 38: 133 - 144.

de Melo JPR, da Camara CAG, Lima GS, Moraes MM, Alves PB. 2018. Acaricidal properties of the essential oil from Aristolochia trilobata and its major constituents against the two-spotted spider mite (Tetranychus urticae). Can J Plant Sci 98: 1342 - 1348. https://doi.org/10.1139/cjps-2018-0163

Dória GAA, Silva WJ, Carvalho GA, Alves PB, Cavalcanti SCH. 2010. A study of the larvicidal activity of two Croton species from northeastern Brazil against Aedes aegypti. Pharm Biol 48: 615 - 620. https://doi.org/10.3109/13880200903222952

Feng R, Isman MB. 1995. Selection for resistance to azadirachtin in the green peach aphid, Myzus persicae. Experientia 51: 831 - 833. https://doi.org/10.1007/bf01922438

Filho JMTA, Araújo LC, Oliveira AP, Guimarães AL, Pacheco AGM, Silva FS, Cavalcanti LS, Lucchese AM, Almeida JRGS, Araújo ECC. 2017. Chemical composition and antibacterial activity of essential oil from leaves of Croton heliotropiifolius in different seasons of the year. Braz J Pharmacog 27: 440 - 444. https://doi.org/10.1016/j.bjp.2017.02.004

Finney DJ. 1971. Probit Analysis, Cambridge University Press, Cambridge, USA.

García M, Donadel OJ, Ardanaz CE, Tonn CE, Sosa ME. 2005. Toxic and repellent effects of Baccharis salicifolia essential oil on Tribolium castaneum. Pest Manage Sci 61: 612 - 618. https://doi.org/10.1002/ps.1028

Hiruma-Lima CA, Gracioso JS, Rodriguez JA, Haun M, Nunes DS, Souza-Brito ARM. 2000. Gastroprotective Effect of Essential Oil from Croton cajucara Benth. (Euphorbiaceae). J Ethnopharmacol 69: 229 - 234. https://doi.org/10.1016/s0378-8741(99)00127-0

Hong T, Perumalsamy H, Jang K, Na E, Ahn YJ. 2018. Ovicidal and larvicidal activity and possible mode of action of phenylpropanoids and ketone identified in Syzygium aromaticum bud against Bradysia procera. Pest Biochem Physiol 145: 29 - 38. https://doi.org/10.1016/j.pestbp.2018.01.003

Koul O, Walia S. 2009. Comparing impacts of plant extracts and pure allelochemicals and implications for pest control. CAB Rev Perspect Agric Vet Sci Nutr Nat Res 49: 1 - 30. https://doi.org/10.1079/pavsnnr20094049

Lima JKA, Albuquerque ELD, Santos ACC, Oliveira AP, Araújo APA, Blank AF, Arrigoni-Blank MF, Alves PB, Santos DA, Bacci L. 2013. Biotoxicity of some plant essential oils against the térmite Nasutitermes corniger (Isoptera: Termitidae). Ind Crop Prod 47: 246 - 251.

https://doi.org/10.1016/j.indcrop.2013.03.018

Lucia A, Audino PG, Seccacini E, Licastro S, Zerba E, Masuh H. 2007. Larvicidal effect of Eucalyptus grandis essential oil and turpentine and their major components on Aedes aegypti larvae. J Am Mosq Control Assoc 23: 299 - 304. https://doi.org/10.2987/8756-971x(2007)23[299:leoege]2.0.co;2

Macêdo DG, Ribeiro DA, Coutinho HD, Menezes I.R, Souza M. 2015. Práticas terapêuticas tradicionais: uso e conhecimento de plantas do cerrado no estado de Pernambuco (Nordeste do Brasil). Bol Latinoam Caribe Plant Med Aromat 14: 491 - 508.

Melo JPR, Da Camara CAG. 2019. Produto formulado a base de óleos essenciais de Citrus reticulata para o controle da traça das crucíferas (Plutella xylostella). BR Patent No. 1020150264488. Instituto Nacional da Propriedade Industrial, Brasilia, Brasil.

Morais SM, Cavalcanti ESB, Bertini LM, Oliveira CLL, Rodrigues JRB, Cardoso JL. 2006. Larvicidal Activity of Essential Oils From Brazilian Croton Species Against Aedes Aegypti L. J Am Mosq Control Assoc 22: 161 - 164. https://doi.org/10.2987/8756-971x(2006)22[161:laoeof]2.0.co;2

Moraes MM, da Camara CAG, dos Santos ML, Fagg CW. 2012. Essential oil Composition of Eugenia langsdorffii O. Berg.: Relationships between some terpenoids and toxicity against Tetranychus urticae. J Brazil Chem Soc 23: 1647 - 1656. https://doi.org/10.1590/s0103-50532012005000029

Moraes MM, Camara CAG, Silva MMC. 2017. Comparative toxicity of essential oil and blends of selected terpenes of Ocotea species from Pernambuco, Brazil, against Tetranychus urticae Koch. Ann Acad Bras Cienc 89: 1417 - 1429. https://doi.org/10.1590/0001-3765201720170139

Mossa ATH. 2016. Green pesticides: Essential oils as biopesticides in insect-pest management. J Environ Sci Technol 9: 354 - 378. https://doi.org/10.3923/jest.2016.354.378

Boletín Latinoamericano y del Caribe de Plantas Medicinales y Aromáticas / 258 
Nascimento AF, da Camara CAG, de Moraes MM. 2012. Essential oil composition and acaricidal activity of Schinus terebinthifolius from atlantic forest of Pernambuco, Brazil against Tetranychus urticae. Nat Prod Commun 7: 129 - 132. https://doi.org/10.1177/1934578x1200700141

Nerio LS, Olivero-Verbel J, Stashenko E. 2010. Repellent activity of essential oils: a review. Bioresour Technol 101: 372 - 378. https://doi.org/10.1016/j.biortech.2009.07.048

Neves IA, da Camara CAG. 2011. Acaricidal Activity against Tetranychus urticae and essential oil composition of four Croton species from Caatinga biome in Northeastern Brazil. Nat Prod Commun 6: 893 - 899. https://doi.org/10.1177/1934578x1100600633

Paluch G, Bartholomay L, Coats J. 2010. Mosquito repellents: a review of chemical structure diversity and olfaction. Pest Manag Sci 66: 925 - 935. https://doi.org/10.1002/ps.1974

Perumalsamy H, Kim NJ, Ahn AJ. 2009 Larvicidal activity of compounds isolated from Asarum heterotropoides against Culex pipiens pallens, Aedes aegypti, and Ochlerotatus togoi (Diptera: Culicidae). J Med Entomol 46: 1420 - 1423. https://doi.org/10.1603/033.046.0624

Pinho-da-Silva L, Mendes-Maia PV, Garcia TMN, Cruz JS, de Morais SM, Coelho-de-Souza AN, Lahlou S, LealCardoso JH. 2010. Croton sonderianus essential oil samples distinctly affect rat airway smooth muscle. Phytomedicine 17: 721 - 725. https://doi.org/10.1016/j.phymed.2010.01.015

Ribeiro N, Camara C, Ramos C. 2016. Toxicity of essential oils of Piper marginatum Jacq. against Tetranychus urticae Koch and Neoseiulus californicus (McGregor). Chil J Agric Res 76: 71 - 76. https://doi.org/10.4067/s0718-58392016000100010

Robertson JL, Jones MM, Olguin E, Alberts B. 2017. Bioassays with arthropods, CRC Press., Boca Raton, USA.

Sato ME, Miyata T, da Silva M, Raga A, de Souza-Filho MF. 2004. Selections for fenpyroximate resistance and susceptibility, and inheritance, cross-resistance and stability of fenpyroximate resistance in Tetranychus urticae Koch (Acari: Tetranychidae). Appl Entomol Zool 39: 293 - 302. https://doi.org/10.1303/aez.2004.293

Silva CGV, Zago HB, Júnior HJGS, da Camara CAG, Oliveira JV, Barros R, Schwartz MOE, Lucena MFA. 2008. Composition and Insecticidal Activity of the Essential Oil of Croton grewioides Baill. against Mexican Bean Weevil (Zabrotes subfasciatus Boheman). J Essent Oil Res 20: 179 - 182. https://doi.org/10.1080/10412905.2008.9699985

Silva CGV, Oliveira JCS, da Camara CAG. 2018. Insecticidal activity of the ethanolic extract from Croton species against Plutella xylostella L. (Lepidoptera: Plutellidae). Rev Fac Nac Agron Medellín 71: 8543 - 8551. https://doi.org/10.15446/rfna.v71n2.62881

Silva JS, Sales M, Gomes APS, Torres DSC. 2010. Sinopse das espécies de Croton L. (Euphorbiaceae) no estado de Pernambuco, Brasil. Acta Bot Bras 24: 441 - 453. https://doi.org/10.1590/s0102-33062010000200015

Souza AVV, Britto D, Santos US, Bispo LP, Turatti ICC, Lopes NP, Oliveira AP, Almeida JRGS. 2017. Influence of season, drying temperature and extraction time on the yield and chemical composition of 'marmeleiro' (Croton sonderianus) essential oil. J Essent Oil Res 29: 76 - 84. https://doi.org/10.1080/10412905.2016.1178183

Van Ee BW, Riina R, Berry PE. 2011. A revised infrageneric classifi cation and molecular phylogeny of New World Croton (Euphorbiaceae). Taxon 60: 791 - 823. https://doi.org/10.1002/tax.603013

Van Leeuwen T, Vontas J, Tsagkarakou A, Dermauw W, Tirry L. 2010. Acaricide resistance mechanisms in the two-spotted spider mite Tetranychus urticae and other important Acari: a review. Insect Biochem Mol Biol 40: 563 - 572. https://doi.org/10.1016/j.ibmb.2010.05.008 